\title{
Circuit
}

Musiques contemporaines

\section{Bruit, son, silence : découvrir ce chant infiniment moderne}

\section{Gilles Tremblay}

Volume 5, numéro 1, 1994

Gilles Tremblay : réflexions

URI : https://id.erudit.org/iderudit/902088ar

DOI : https://doi.org/10.7202/902088ar

Aller au sommaire du numéro

Éditeur(s)

Les Presses de l'Université de Montréal

ISSN

1183-1693 (imprimé)

1488-9692 (numérique)

Découvrir la revue

Citer ce document

Tremblay, G. (1994). Bruit, son, silence : découvrir ce chant infiniment moderne. Circuit, 5(1), 37-46. https://doi.org/10.7202/902088ar d'utilisation que vous pouvez consulter en ligne.

https://apropos.erudit.org/fr/usagers/politique-dutilisation/ 


\section{Découvrir ce chant infiniment moderne}

Paru sous le titre "Le point de vue d'un compositeur», Vie spirituelle, Paris, mars-avril 1974, pp. 234-245. Version révisée in Communauté chrétienne, $n^{\circ} 144$, novembre-décembre 1985 sous le titre retenu ici.

Lorsque Benoît Lacroix et Michel Giard m'ont demandé un article pour Communauté Chrétienne, un texte élaboré il y a quelques années pour la vie spirituelle m'est immédiatement venu à l'esprit car il traitait précisément des mêmes questions posées aujourd'hui. Comme la situation n'a guère changé, dans la francophonie du moins, sa version révisée a servi de base au présent article. Il avait été suscité par le Frère André Gouzes, o.p., maintenant directeur d'un centre fort vivant de musique liturgique à l'Abbaye de Sylvanès en France, et provoqué sous forme de dialogue, par les questions de dom André Laberge, bénédictin, et de l'abbé Lamoureux, de l'archevêché de Montréal.

Permettez-moi de commencer par vous livrer, en bref, ce que j'appellerais volontiers mon credo musical. II faut constater une détérioration extrême de la musique dans l'Église, devenue sorte de lie allant du néo-Moyen Âge au musichall en passant par toute la gamme des intermédiaires. Après cette constatation, il devient difficile sinon étouffant pour le musicien, aujourd'hui, de travailler dans cette ambiance. Je m'exprime ici de façon extrême, mais je crois qu'il faut le faire. Il faut balayer cette production, complètement. Même si, exceptionnellement, quelques éléments peuvent être bons, le poids en est si lourd que ma réaction est de faire table rase d'une production qui, à mon avis, ne reflète rien d'autre qu'une esthétique musicale de société de consommation dont la pastorale est réduite à une démagogie où le peuple de Dieu serait considéré comme une * clientèle ».

C'est l'aspect négatif de la question que je viens d'évoquer. Allons au plus vite à la recherche de l'aspect positif. II se trouve, du moins je le pense, dans la découverte du plain-chant. Non pas dans le retour - j'ai bien employé le mot découverte et non « redécouverte *-, car le plain-chant des paroisses autrefois, ici, au Québec, était - pas toujours mais souvent - très médiocre. Donc, découverte du plain-chant, parce que le plain-chant est à la fois la musique la plus belle, la plus actuelle, la plus vivante et la plus libre qui soit. Débordante d'une richesse infinie par les signes, symboles, équilibres, formes, mouvements qui la composent comme issus de la vie, c'est également la musique la plus liturgique qui soit. Car elle évite le piège d'une complaisance 
en elle-même, en sa propre beauté, qui l'enfermerait dans ses propres limites. Au contraire, elle est toute tournée vers la lovange, elle en est pour ainsi dire illuminée. Musique transfigurée par l'objet de sa louange. Elle passe ainsi, elle se transmute à un autre plan de beauté, de beauté infinie, enveloppante et émanant de Celui qu'elle chante.

Je ne crois pas, cependant, au plain-chant grégorien comme à une chose gelée, figée, interdisant toute autre création musicale. Je crois, au contraire, à sa présence comme à une véritable sève spirituelle et musicale, sève spirituelle et musicale qui est en même temps exemplaire, à partir de laquelle on peut commenter, développer, extrapoler avec le langage musical le plus évolué. Celui de notre époque. Ce pourrait être dans une optique très moderne, des neumes d'espace, des neumes de timbre, des neumes de tempi, des neumes de densité, qui auraient un rapport avec les textes originaux du plain-chant, que l'on pourrait traiter de façon très élargie, comme tous les compositeurs de l'Histoire ont traité les cantus firmus, que ce soit Palestrina, Josquin Després, Monteverdi. II ne s'agit pas de traiter le plain-chant comme un cantus firmus uniquement mais de le considérer comme une source, comme un point de repère autour duquel on fait des gloses ou des tropes, pour employer un vocabulaire du Moyen Âge cher à Pierre Boulez.

II me paraît assez significatif que les meilleurs musiciens de l'heure soient absents d'une élaboration musicale liturgique. Un travail rationnel en ce sens doit être fait, en collaboration également avec les meilleurs écrivains. C'est très important. Car la suppression du latin - je ne suis nullement un puriste fanatique! - pose certains problèmes prosodiques et musicaux réels, mais tout à fait surmontables. Dans ce domaine, je ne crois pas du tout à l'amateurisme, tant dans le domaine littéraire que dans le domaine musical, c'est-à-dire qu'il faudrait vraiment de grands écrivains, de grands poètes pour les textes chantés qui travailleraient, bien sûr, en collaboration avec les musiciens.

Vous avez parlé d'une dégradation irrémédiable de la musique dans l'Église.

Je n'idéalise pas du tout ce qui se faisait dans le passé comme si c'était supérieur à ce qui se fait maintenant, mais il est sûr que l'Église a été pendant plusieurs siècles le foyer de la culture humaine, un foyer extraordinaire, en architecture, en peinture, en sculpture, en musique, et qu'il y a eu depuis, une espèce d'éclatement. Depuis la Renaissance, il y a eu une dégradation non pas des œuvres, non pas de la musique, mais du sens liturgique que peut avoir la musique. 
Tout à l'heure je disais, à propos du plain-chant, que c'est une musique de louange qui n'est pas fermée sur sa propre beauté. Un exemple: je suis un très grand admirateur de Mozart. Or, je ne trouve pas que les Messes de Mozart soient vraiment des musiques liturgiques. Je ne dis pas sacrées parce que, pour moi, toute création est sacrée, et je trouve les Opéras de Mozart aussi sacrés que ses Messes. II s'agit d'objets qui sont très beaux en euxmêmes. Dans la musique grégorienne dite de plain-chant, la musique est également très belle, mais c'est une musique extrêmement humble, c'est une musique de lovange qui ne fait pas écran devant Celui qu'elle louange! A ce moment-là, et c'est peut-être cela qui s'est perdu, cette musique de louange reflète Celui qu'elle louange. On assiste à une transfiguration: l'acte de louange est transformé par Celui qu'il louange.

Vous voulez dire qu'elle est l'expression personnelle de celui qui est en adoration, mais que dans son élan elle devient la révélation de celui qu'elle love?

C'est-à-dire qu'elle reste tout à fait personnelle, mais la louange devient transparente. Elle est augmentée par transfiguration, par reflet, par illumination de celui qu'elle louange. Tandis que l'œuvre qui se referme sur elle-même peut avoir une beauté extraordinaire, mais tout s'arrête là. Un extrait du livre de la Sagesse (13: 1-9) est éclairant à ce sujet:

Oui, vains par nature tous les hommes en qui se trouvait l'ignorance de Dieu, qui, en partant des biens invisibles, n'ont pas été capables de connaître Celui-quiest,

et qui, en considérant les œuvres, n'ont pas reconnu l'Artisan.

Mais c'est le feu, ou le vent, ou l'air rapide,

ou la voûte étoilée, ou l'eau impétueuse,

ou les luminaires du ciel, qu'ils ont considérés comme des dieux, gouverneurs du monde!

Que si, charmés de leur beauté, ils les ont pris pour des dieux, qu'ils sachent combien leur Maître est supérieur, car c'est la source même de la beauté qui les a créés.

Et si c'est leur puissance et leur activité qui les ont frappés,

qu'ils en déduisent combien plus puissant est Celui qui les a formés,

car la grandeur et la beauté des créatures

font, par analogie, penser à leur Auteur.

Ceux-ci toutefois ne méritent qu'un blâme léger;

peut-être en effet ne s'égarent-ils qu'en cherchant Dieu et en voulant le trouver:

versés dans ses œuvres, ils les explorent

et se laissent prendre aux apparences, tant ce qu'on voit est beauté! 
Et pourtant eux non plus ne sont point pardonnables:

sils ont été capables d'acquérir assez de science pour pouvoir scruter le monde,

comment n'en ont-ils pas plus tôt découvert le Maître!

J'ai rarement vu un texte aussi actuel. Car, qu'est-ce qui se passe dans les œuvres humaines? Elles (cela paraît prétentieux, mais i'y crois beaucoup) sont comme une participation à la création. C'est même pour cela que l'on peut dire que toute création est sacrée : elle vient du même mouvement. Mais se pose pour elle le même problème: sera-telle écran ou sera-telle cristal $己$

Ne retrouve-ton pas, de façon semblable, deux mouvements dans le besoin de chanter?

Autrefois, on était sensible au fait que l'on chantait devant Dieu, on chantait pour lui ou on le chantait. Alors que maintenant, j'ai l'impression que l'on chante, pour une part, les uns pour les autres, dans un souci de se sentir en communion, si bien que la musique est devenue un moyen de rassemblement.

Je ne crois pas que ceci soit nouveau. C'est enraciné dans les profondeurs de l'homme. Ce sentiment de se sentir un, pris en un, on le retrouve dans tout chant à l'unisson et dans des religions tout à fait étrangères à la nôtre.

Par exemple, dans certaines musiques bouddhiques coréennes, on a des unissons qui se défont, mais qui reviennent toujours à se refondre ensemble. Je crois qu'il s'agit là d'un des grands instincts humains et je crois que cela mériterait d'être expérimenté. Songez à la fameuse syllabe des Hindous, la syllabe sacrée, la syllabe créatrice, celle qui contient toutes les harmoniques sur un seul son que l'on chante à l'unisson.

\section{Et que pensez-vous du chant choral luthérien?}

Je connais le chant luthérien surtout du point de vue musical. J'ai assisté à quelques offices en Allemagne; c'était très impressionnant. Je ne crois pas cependant que ce chant ait la valeur et l'envergure du plain-chant, lequel est infiniment plus libre sur le plan rythmique.

Quand je parle de musique vivante et très libre, je pense vraiment à une musique en progrès. Ce qu'il y a d'extraordinaire dans la rythmique du plainchant qui a rejoint les plus grands rythmiciens de l'Histoire, notamment les musiciens du vingtième siècle, Debussy, Stravinsky, Messiaen, etc., c'est justement cette vie rythmique où le rythme est un mouvement en perpétuelle 
transformation. Cela demande un plus grand effort rythmique, mais aussi cela coule davantage, et plus librement.

Comment pensez-vous que les compositeurs puissent travailler actuellement?

Actuellement, le travail ne peut pas être intéressant pour eux. Personnellement, je n'ai aucune envie de composer des petits cantiques alors que la matière que j'utilise dans les œuvres instrumentales profanes me paraît infiniment plus religieuse, au sens le plus sacré, car elle est participation à la création. J'ai l'impression que c'est plus important de composer en ce sens maintenant. Surtout sachant que nous avons déjà un répertoire d'une richesse infinie qui dort. La première chose à faire, c'est de ne pas ajouter au fatras, mais plutôt de nettoyer le répertoire, de faire un grand ménage et de découvrir cette fraîcheur, ce chant infiniment moderne. À partir de là, ensuite, on pourra greffer et faire toutes les expériences possibles, même les plus délirantes! Sans aucune dictature, bien sûr. Un peu comme Philippe de Néri l'a fait en instituant ses séances de l'Oratoire où l'on trouvait des paraliturgies et des homélies auxquelles il invitait les grands orateurs et les grands musiciens de l'époque (n'oubliez pas que l'Oratoire a donné le nom aux oratorios). $C^{\prime}$ était très expérimental. Ce devait être amusant et très poétique. Ce n'était pas de la liturgie proprement dite, mais ce pourrait être un moyen pour que le public et les artistes eux-mêmes se sentent très libres et redécouvrent la musique sacrée.

Quand je parle de balayage, je schématise un peu, mais pourquoi pas une période de jeûne qui permettrait ensuite de mûrir quelque chose? Cela ferait peutêtre découvrir des choses essentielles, essentielles à la musique et essentielles à la prière, comme le silence. Car le silence est pour moi la condition première et il est peut-être la musique première. En outre, il y a dans la liturgie une chose qui est encore plus importante que la musique et que cela fera peutêtre découvrir: c'est la parole. II faut pouvoir proclamer la parole dignement, et clairement, de façon belle. Avec parole et silence, vous avez des éléments musicaux. Une lecture bien faite, c'est déjà une lecture qui est déjà très musicale. Quand les gens auront découvert un certain sens de l'authenticité, ils pourront alors immédiatement chanter certaines hymnes de plainchant, à condition que quelqu'un les leur enseigne. Cela me paraît finalement très simple...

II faut quand même un maître de chapelle?

Oui. II faut qu'il ait, lui, cette culture. Je ne pense pas que les goûts soient l'apanage de quelques-uns, et c'est vrai qu'il y a des gens qui ont du goût et 
qu'il y en a d'autres qui n'en ont pas. Mais les gens qui ont du goût ne sont souvent pas ceux qu'on pense. Et quand je parle de culture, je ne parle pas du tout de culture encyclopédique. Je pense que les enfants, par exemple, ont un goût assez inné. Les milieux que l'on dit populaires ont aussi un goût inné, seulement on ne leur fournit jamais l'occasion de l'exercer. Au contraire, le peuple est noyé la plupart du temps dans une culture mercantile que l'on baptise populaire alors qu'elle ne vient pas du peuple, ce qui est une gigantesque imposture. Là encore, en ce désert, le rôle de l'Église pourrait être très grand, à la mesure de la soif spirituelle de l'homme de notre époque, car si le rôle premier de l'Évangile n'est évidemment pas l'esthétique, on peut difficilement, il me semble, dissocier amour, spiritualité et beauté. Et la crainte de l'esthétisme n'a-t-elle pas été faussée parfois en horreur de la beauté?

Si le plain-chant est une découverte, comment voyez-vous qu'il puisse inspirer à nouveau des compositeurs?

Pour répondre très précisément, il faudrait mûrir la question pour arriver à des solutions qui, d'ailleurs, resteraient personnelles. Ce que je veux dire, c'est que la présence de ce plain-chant s'imposerait aux compositeurs comme un point de repère et serait en même temps exemplaire. Et ji'y verrais combinées les expressions musicales de notre époque. Je pense qu'on peut très bien garder le plain-chant tel quel, en faire des gloses, des tropes, faire des parenthèses si vous voulez, qui soient tout à fait de notre époque. Peut-être pourrait-on abandonner plus tard ce qui aura été tenté, quand on aura fait quelque chose de mieux. Mais en attendant, le principe de greffes, de boutures pour parler en langage de jardinier, semble le plus vivant et peut-être le plus enthousiasmant aussi. J'y verrais d'ailleurs - et je le souhaiterais - les plus grands musiciens de notre époque y travailler, avec les meilleurs artistes. Pourquoi ne pas leur faire des commandes?

Ne craignez-vous pas tout de même qu'en proposant la découverte du plainchant, on ne vous accuse de vouloir en rester au passé?

Je ne considère pas du tout que c'est parce qu'une œuvre a été conçue dans le passé qu'elle doit être considérée comme un objet de musée, c'està-dire un objet mort, ou à peu près, si du moins cette œuvre-là a une action, c'est-à-dire me frappe. Je trouve, par exemple, que Mozart est un des musiciens les plus modernes qui soient. Donnons des raisons techniques: les concertos de Mozart, par exemple, au point de vue forme, vont beaucoup plus loin que la plupart des musiques contemporaines. On y trouve une espèce de point extrême dans la fantaisie de la forme, extrême de perfection et de vie. 
Quand on les joue aujourd'hui, on ne s'y réfère pas comme à des objets du passé : ils vivent dans le temps présent.

Quand on apporte l'objection à laquelle vous faites allusion au sujet du plain-chant, je pense qu'on est victime de cette espèce de dualisme très occidental où l'on oppose un élément contre l'autre et le passé au présent et même au futur. Je parle peut-être en poète, mais je crois personnellement à une simultanéité, à une fraternité de tous les temps dans un présent vivant.

Pour revenir au plain-chant d'une façon plus technique, je souligne que ce plain-chant contient des éléments qui sont ceux avec lesquels une grande partie de la terre s'exprime. Ef là où je trouve sa musique extrêmement moderne, c'est qu'on la redécouvre un peu comme certains textes bibliques, par exemple, certains textes d'Ezéchiel. Messiaen aime citer Ezéchiel écrivant: "Les étoiles chantent», et il ajoute: "C'est extraordinaire de lire cette phrase aujourd'hui où nous savons justement que les étoiles envoient des radiations que l'on pourrait traduire en chant. » « Les étoiles chantent », c'est vraiment prophétique et très actuel. De la même façon les recherches contemporaines, dans le domaine rythmique entre autres (métrique variable), éclairent les mélodies grégoriennes d'un jour tout à fait neuf, surtout si elles sont exécutées sans traîner. On leur découvre des qualités extrêmement jeunes et remplies de vie, et un caractère d'universalité surprenant. Je pense en particulier au magnifique Gloria XV sur quatre sons, aux accents à la fois hindous et africains.

Voici encore un autre point que j'aimerais développer: les mélodies de plain-chant ont été notées au moyen de neumes ou, si vous préférez, au moyen de contours, de mouvements. Or, on touche ici à une symbolique très forte et qui est de tous les temps, la symbolique des mouvements. Tout art musical, comme disaient les Grecs, est fait de mouvements, et si l'on enlève les sons, on a encore cette espèce d'écorce, de squelette de mouvement qui est extrêmement stimulant pour le musicien d'aujourd'hui. C'est pourquoi, au tout début de notre entretien, j'ai parlé de neumes d'espaces, de neumes de densité même. J'ai déjà fait allusion à certaines grandes expériences de musique contemporaine.

Pour la densité, je pense notamment à Xenakis et à la théorie des gaz appliquée à la musique, où la densité des événements varie constamment, un peu comme la pluie dans la nature. On pourrait, par exemple, partir des neumes, suivre leurs courbes en les transformant en intensité, densité et même espace, les mouvements spaciaux épousant leurs formes. Imaginez la foison de rapports mélodiques et symboliques mis en action! Sommes-nous en 
Utopie ¿ Peut-être. Personnellement, je la trouve extrêmement stimulante et inspiratrice. Dans le plain-chant, tous ces neumes, toutes ces mélodies, ne sont encombrés de rien, ce qui donne justement cet aspect dégagé et très libre. C'est une musique qui, mélodiquement, ne peut presque pas aller plus loin.

On trouve rarement, dans une musique harmonique, une liberté mélodique aussi grande. Même les Fugues de Bach, qui sont belles, n'ont pas cette liberté mélodique du chant grégorien. C'est un chant libre, libre de tout. J'emploie le mot libre en ce sens que c'est une musique nue, qui ne croule pas sous un fatras de vêtements, ni sous de lourds carcans.

Quel est dans l'avenir immédiat de la musique liturgique, votre quotient de confiance dans la grâce des musiciens et des croyants?

Si j'analyse la situation telle qu'elle est maintenant, je vous répondrai sincèrement: je ne suis pas du tout optimiste. Si je veux vraiment être rationnel, être optimiste serait de l'inconscience. Mais, au fond de moi-même - je ne fais pas toujours des raisonnements de type rationnel - , je crois beaucoup à l'imprévisible. Dans la vie, il y a toujours des événements imprévisibles. Certains signes précurseurs font qu'il n'y a pas de quoi être pessimiste et je crois que ces signes sont suffisants pour être très optimiste. Mais, encore une fois, c'est dans une optique d'imprévisible.

Devant la situation actuelle, je ne me cogne pas du tout la tête contre les murs. D'abord parce que ça ne sert à rien et puis parce que je crois que l'expression spirituelle - il y a un mot qu'on emploie très peu aujourd'hui, la grâce - ça s'exprime par toutes sortes de moyens inattendus et il y a peut-être toute une partie de l'expression spirituelle qui se trouve déplacée ailleurs. Je souhaite qu'elle revienne dans l'Église, pour le plus grand bien de l'Église et de la communauté chrétienne.

En guise de conclusion d'ordre pratique et à titre indicatif je verrais, à partir de la situation actuelle, une démarche en quatre directions:

- L'insertion, avec l'aide concrète de l'Église (au niveau diocésain, national ou international), d'un répertoire des œuvres grégoriennes chantables par la foule, sorte d'opération de ressourcement musical et religieux dans la beauté, en vue de la liturgie.

- L'organisation de concerts de musique sacrée sur des grands textes religieux, avec des commandes aux meilleurs compositeurs de notre temps. Soyons clairs, cela exclut les "auteurs-compositeurs " de type mercantiloamateurs, même parmi les plus connus. - Ces concerts sacrés devraient 


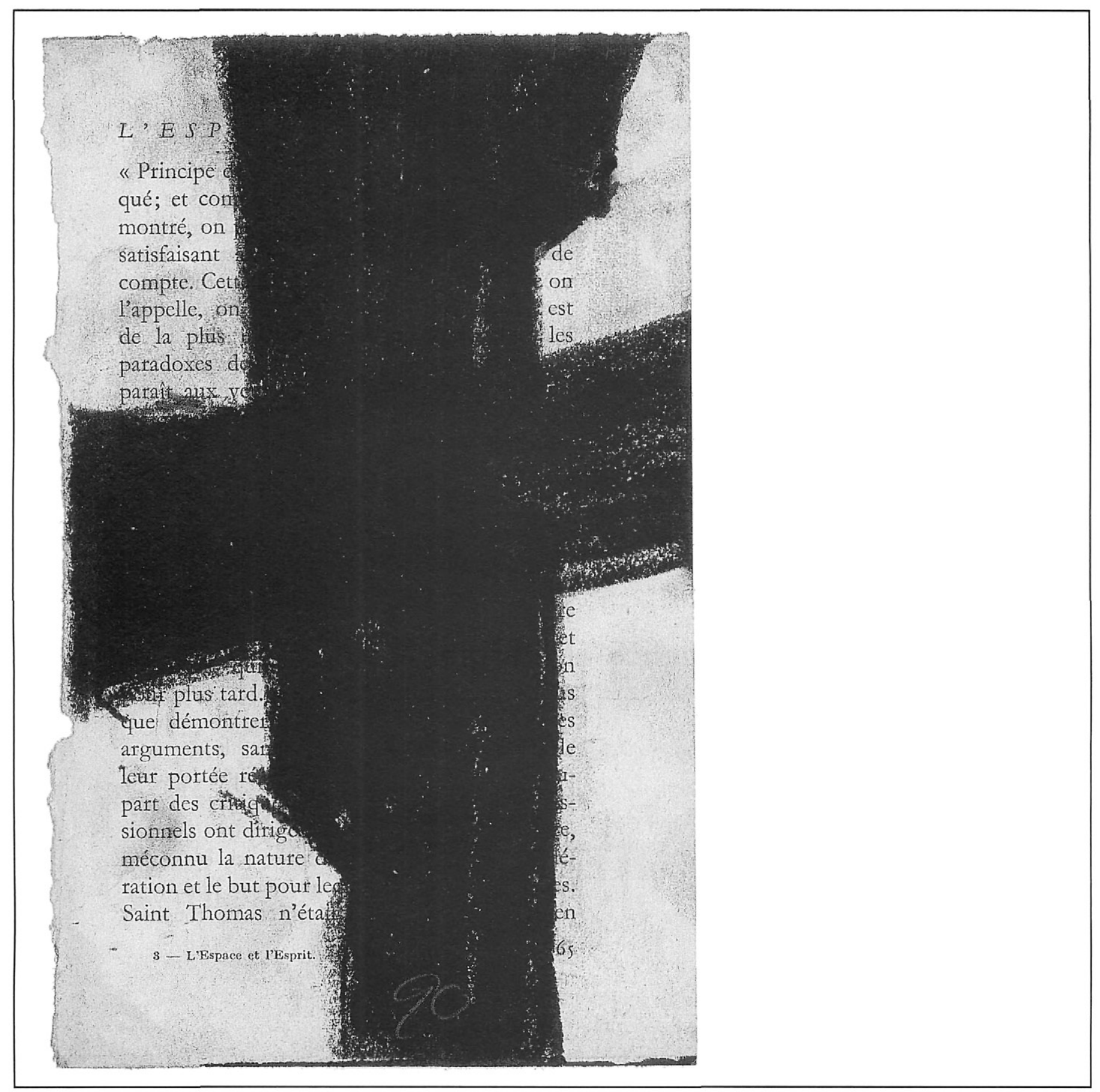


s'inspirer de l'esprit de Philippe de Néri, et supposent la collaboration entre compositeurs, poètes et théologiens.

- La formation artistique des futurs responsables en l'Église, prêtres ou laïcs, au même titre que la formation théologique, car l'art est une des formes de la parole.

- La création, au niveau épiscopal, d'une commission de musique sacrée, composée de professionnels issus des ressources du milieu (1), avec un mandat très large et un appui financier significatif, chargée de l'élaboration globale de la musique dans l'Église.

J'ai employé le mot «beauté " dans cet article. Mot tabou s'il en est - le questionnaire que Communauté chrétienne m'a envoyé ne l'emploie pas une seule fois, ce qui est fort prudent - car on ne peut définir la beauté de façon satisfaisante, comme les efforts des philosophes en témoignent. Je tiens à préciser que je suis moi aussi fort dépourvu (comme la cigale, une musicienne également) de définition à son égard. Même si elle ne se définit pas, nous signifiant par là qu'on ne peut la posséder, lorsqu'elle nous est manifestée nous savons très bien la reconnaître. Pendant que j'écris ces lignes, des iris bleus éclosent.

Plénitude du vers du psalmiste : «De tout votre art louez le Seigneur... »
(1) Je pense aux efforts très positifs effectués en ce sens déjà dans quelques endroits. 